\title{
Canada behind in regulating farmyard antibiotics
}

$\mathrm{N}$ ew restrictions on the use of antibiotics on farm animals in both the United States and Canada may help mitigate risks to human health due to antibiotic resistance. However serious concerns remain - particularly in Canada. According to the Ontario Medical Association (OMA), Canada is trailing the US on control of antibiotic use on farms.

"It's really unusual that Canada is lagging so far behind," says Dr. Doug Weir, OMA's past-president and its spokesperson on antibiotic resistance. "We'd like to see tougher legislation." Both Canada and the US should follow Europe's example by enacting comprehensive bans on nontherapeutic usage of antibiotics in animal husbandry, he says.

The US recently passed far-reaching measures to curb the nonmedical use of antibiotics in farming, but Canada is taking a piecemeal approach that lacks government enforcement. In December 2013, the Chicken Farmers of Canada, which regulates 2700 chicken farmers in conjunction with provincial chicken marketing boards, announced it will eliminate preventive use of medically important antibiotics, including cephalosporins and fluoroquinolones, by May 2014.

Fluoroquinolones are routinely used in chicken's drinking water and cephalosporin is injected into eggs. Both practices are already banned in the US. In addition to these steps, the farmers group is mandating veterinary supervision of all drugs that Health Canada has designated medically critical.

However, these are industry rules, not government regulations, and the government will not be enforcing them. Instead, the Chicken Farmers of Canada will insist on compliance, says Steve Leech, who manages food safety, animal care and research for the association. "It will be enforced through our on-farm safety program."

But the public will have no way of knowing whether that's being done; as Leech acknowledges, his industry does not release information on its enforcement activities.

While this initiative is positive, Weir says self-regulation may not be sufficient.

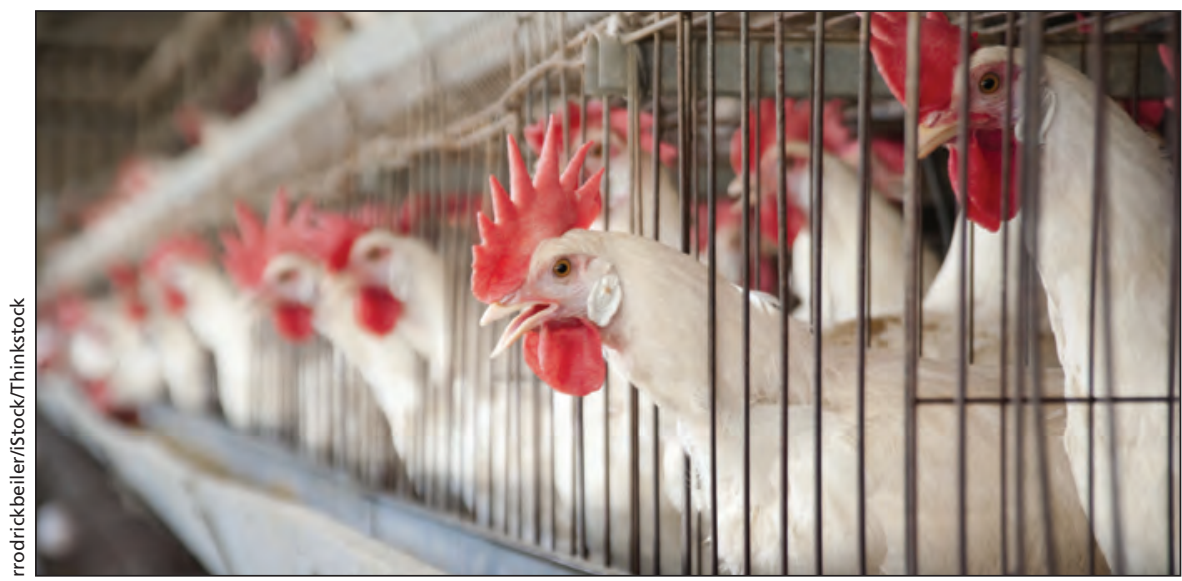

New industry regulations will ban the use of fluoroquinolones, which are routinely used in chicken's drinking water, and cephalosporin, which is injected into eggs.

Dr. Lynora Saxinger, chair of the Antimicrobial Stewardship and Resistance Committee of the Association of Medical Microbiology and Infectious Disease Canada, agrees. Action from the federal government is long overdue, she says. "There is a lack of regulatory clarity. Ottawa has certainly studied the issue long enough."

Saxinger is particularly concerned about loopholes in federal laws that allow large volumes of antibiotics to be imported and used on animals without surveillance or regulation, a problem the OMA recently rebuked. "We strongly urge the federal government to curtail these loopholes," says Weir.

According to the Public Health Agency of Canada (PHAC), more than three-quarters of all antimicrobials consumed in Canada are used in animals, and approximately $90 \%$ of these are used to promote growth or to prophylactically guard against disease and infection. PHAC is concerned about resistance levels to the third-generation cephalosporins such as ceftiofur and ceftriaxone, and the fluoroquinolones such as enrofloxacin and ciprofloxacin detected in bacteria found on retail meat and poultry, says PHAC spokesman Stephane Shank.

He says $40 \%$ of samples taken from retail poultry in British Colombia in 2012 contained bacteria that were resistant to cephalosporins. In Ontario and Quebec, about 30\% of samples showed resistance to cephalosporins.

In contrast to upcoming self-regulation measures in Canada, the US is sys- tematically limiting farmyard antibiotic use. On Dec. 11, 2013, the US Food and Drug Administration (FDA) instructed pharmaceutical companies to voluntarily remove animal "growth enhancement" indications from medically important drugs including cephalosporins, penicillins and fluoroquinolones. This means the nontherapeutic use of these antibiotics will be stopped.

The US agricultural industry uses an estimated $80 \%$ of all antibiotics in the country. More than 2 million Americans suffer from drug-resistant infections annually, resulting in 23000 deaths, according to the US Centers for Disease Control and Prevention.

This latest regulation is the closest the US government can get to "pulling the drugs," says Morgan Scott, a veterinary professor at Kansas State University College of Veterinary Medicine in Manhattan, Kansas. "The FDA cannot act to ban drug uses unless it can prove harm, which is extremely difficult," says Scott, "Instead they can say that certain uses are imprudent."

Not everyone thinks the FDA is being aggressive enough. Congresswoman Louise Slaughter, a microbiologist who proposes legislation to protect medically important antibiotics, says it's an "inadequate response to the overuse of antibiotics on the farm with no mechanism for enforcement and no metric for success." - Paul Christopher Webster, Toronto, Ont.

CMAJ 2014. DOI:10.1503/cmaj.109-4698 